\title{
Estudio acerca de los factores que influyen en la pérdida de interés hacia las matemáticas en los estudiantes del colegio Filipense de la ciudad de San Juan de Pasto, Colombia.
}

Fabián Leonardo Muñoz Muñoz, María Janeth Bravo Montenegro, Hilbert Blanco Álvarez

\section{Resumen}

Este artículo presenta los resultados de una investigación sobre la pérdida gradual del interés hacia las matemáticas de 163 estudiantes del Colegio Filipense "Nuestra Señora de la Esperanza" de la ciudad de San Juan de Pasto (Nariño). Los datos se recolectaronpor medio de un cuestionario tipo Likert y entrevistassemiestructuradas. Finalmente, se concluyó que la pérdida del interés hacia esta disciplina se debe a varios motivos: la desaparición paulatina del juego y de actividades didácticas que en la educación básica tenían una mayor presencia, la falta de actividades de motivación por parte del profesor y la poca relación que los estudiantes de la educación media ven a los temas tratados en clase de matemáticas con la vida diaria.

Palabras Claves: Actitud por las matemáticas, Influencias sociales, Motivación

\section{Summary}

Gradual This article presents the results of an investigation on the loss of the interest towards the mathematics of 163 students of the College Filipense "Our Lady of the Hope "of the city of San Juan of Pasture (Nariño). The information gathered for way of to questionnaire type Likert and interviewed semistructured. Finally, one concluded that the loss of the interest towards this disciplines owes to several motivate: the responses between the epistle and the gospel disappearance of the game and of didactic activities that in the basic education had to major presence, the lack of activities of motivation on the part of the teacher and small relation that the students of the education it happens eats to the topics treated in class of mathematics with the daily life.

Keywords: Attitude for the mathematics, social Influences, Motivation.

\section{Abstract}

Thispaper presents the resultsofan investigation ofthe gradual lossofinterest inmathematicsof163 studentsof the ColegioFilipense "NuestraSeñora de la Esperanza" ofthe city ofSanJuan dePasto(Nariño). Data were collectedusing a Likert questionnaire and semistructured interviews. Finally, it was concluded that the

1 Docente Universidad de Nariño, San Juan de Pasto. Colombia. hilbla@yahoo.com 
lossofinterest inthis subjectis due to severalreasons: the gradual disappearanceof play andlearning activitiesinbasic educationhad a greaterpresence, lack of motivation activities on the partof the professorandlittle relationtothestudentsof secondary educationarethe topics coveredin math classwith daily life.

Keywords: Attitude in mathematics, Social influences, Motivation

\section{Introducción}

$\mathrm{L}$ a investigación de los factores que influyen en la pérdida del interés hacia las matemáticas ha sido tema de interés para los investigadores de los aspectos socioculturales de la educación matemática (Gómez Chacón, 2000; Bishop, 2005; Muñoz \& Bravo, 2007). Esta investigación se centra en indagar la influencia de factores externos como los profesores y compañeros y factores internos como la capacidad matemática y la importancia de esta disciplina para su vida diaria y futuraen el interés que dan a las matemáticas los estudiantes del Colegio Filipense desde el grado $1^{\circ}$ de la educación básica hasta el grado $11^{\circ}$ de la educación media.

Así, entonces se plantea la pregunta de investigación: ipor qué los estudiantes pierden el interés hacia las matemáticas a lo largo de su vida estudiantil?

\section{Materiales y métodos}

Esta investigación fue de tipo descriptiva, e hizo uso de una metodología mixta: cuantitativa y cualitativa, puesto que se aplicó un cuestionario cerrado con escala Likert, al considerarse uno de los métodos más conocidos para medir, por escalas, las actitudes (Ursini, Sánchez, \& Orendain, 2004). La validez de dicho cuestionariose realizó con una prueba piloto, luego se hizo validación por jueces, y al aplicar el cálculo del alfa de Conbrach y el coeficiente de correlación Pearson por mitades, se concluyó que la escala tenía consistencia interna, esto significa que el cuestionario aplicadoproporciona una representación adecuada de la actitud de los estudiantes.

Además, se aplicaron entrevistas con el propósito de profundizar en las ideas de los estudiantes sobre la pérdida de interés hacia las matemáticas y obtener información cualitativa para luego triangularla con los resultados de la escala Likert.

El grupo que participó en la investigación fue de 163 estudiantes de la educación básica y media del Colegio Filipense "Nuestra Señora de la Esperanza" de la ciudad de San Juan de Pasto (Nariño), conformado por 124 mujeres y 39 hombres, con edades que oscilan entre los 5 y 18años.La selección del grupo fue por conveniencia.

\section{Análisis de Datos}

Para realizar el análisis de las respuestas al cuestionario tipo Likert, primero se separaron los cuestionarios en dos niveles: Nivel 1. Estudiantes de la educación básica; Nivel 2. Estudiantes de la educación media. Posteriormente, se cargaron las respuestas en el programa SPSS para ver los porcentajes de puntuación de los valores de la escala en cada uno de los ítems. Luego, las respuestas se analizaron teniendo en cuenta las categorías Profesores, Compañeros, Capacidad matemática e Importancia.

Para realizar el análisis de las entrevistas, se realizaron varias lecturas de las respuestas, a la vez que se iban clasificando de acuerdo a las categorías ya señaladas y el nivel. Dichas categorías y su descripción se presentan en la tabla 1. 


\begin{tabular}{|l|l|l|}
\hline Factores & Categoría & Descripción \\
\hline Internos & $\begin{array}{l}\text { Importan- } \\
\text { cia }\end{array}$ & $\begin{array}{l}\text { Se refiere a la importancia que el estudiante presta al } \\
\text { área de matemáticas; verificando la pertinencia de la } \\
\text { aplicación de esta en su vida diaria, además del posible } \\
\text { uso en su futuro profesional y laboral. }\end{array}$ \\
\cline { 2 - 4 } & $\begin{array}{l}\text { Capacidad } \\
\text { matemática }\end{array}$ & $\begin{array}{l}\text { Trata sobre la capacidad que los estudiantes creen tener } \\
\text { para el desarrollo y la práctica de las matemáticas. }\end{array}$ \\
\hline Externos & Profesor & $\begin{array}{l}\text { En esta categoría se pretende analizar las actitudes que } \\
\text { tienen los estudiantes frente a su profesor de matemáti- } \\
\text { cas, y cómo esto influye en el proceso matemático. }\end{array}$ \\
\cline { 2 - 4 } & $\begin{array}{l}\text { compañe- } \\
\text { ros }\end{array}$ & $\begin{array}{l}\text { Se refiere a las relaciones entre el estudiante y los com- } \\
\text { pañeros, en cuanto al apoyo recibido, atención en clases, } \\
\text { la explicación de temas complejos. }\end{array}$ \\
\hline
\end{tabular}

Tabla 1. Factor, categorías y descripción de cada una de ellas

Análisis de la categoría importancia. Se evidenció que los estudiantes de primer nivel tienen una actitud desfavorable del $27.6 \%$, la cual se incrementa en los estudiantes de segundo nivel a un $34.3 \%$. Es decir, a medida que se avanza en los niveles de aprendizaje son más los estudiantes que adquieren una actitud desfavorable frente a la importancia de esta disciplina en la cotidianidad y para su futuro profesional.

\section{Análisis de la Categoría Capacidad Matemáti-} ca. En esta categoría se pudo observar que la actitud positiva que tienen los estudiantes frente a su capacidad para la práctica de las matemáticas presenta irregularidad, ya que en el primer nivel, donde los estudiantes reciben las primeras bases fundamentales para el área como lo son la suma, resta, multiplicación y división, son quienes se consideran más aptos para las matemáticas con un $55.0 \%$ dan muestra que los niños de seis a ocho años aproximadamente, son más vulnerables a las percepciones que captan del medio donde interactúan, por lo que las motivaciones que reciben permiten que definan de mejor manera su capacidad matemática.

En cuanto a los estudiantes del nivel dos, un 30.7\% manifiestan confianza ante su habilidades matemáticas. De aquí que la actitud hacia la capacidad para las matemáticas sea muy baja en comparación con los estudiantes del nivel uno.
Una entrevista a una estudiante del grado undécimo ilustra lo anterior:

- Entrevistador: iA ti te gustan las matemáticas?

- Estudiante: algunas veces cuando son entendibles

- Entrevistador: ¿Cómo así?

- Estudiante: Simple, si me va bien en los exámenes de matemáticas porque obviamente entiendo, entonces si me gustan.

- Entrevistador: iY si no te va bien?

- Estudiante: No sé, me da mucha rabia porque por más que estudie no me entran y por eso ya no me empiezan a gustar, me parecen complicadas y aburridas.

Análisis de la categoría Profesor. En los resultados de la encuesta se apreció que en el primer nivel, el $68.5 \%$ de los estudiantes respondieron que su profesor influye de una manera positiva en su actitud matemática, porque éste maneja una dinámica de su enseñanza, por tratar con niños de edades que oscilan entre los 6 y 11 años, tiende a ser más creativo en el sentido de crear espacios como el laboratorio de matemáticas; haciendo énfasis en que una de las mejores maneras de aprender 
y practicar las matemáticas es jugando, así como también es una enseñanza más afectiva, ya que los profesores tienen una relación mucho más expresiva, flexible y sensible con sus estudiantes, lo que implica que el proceso de aprendizaje matemático para esta población se vea aún más afectado por el desempeño, motivación y reciprocidad con el profesor.

En el caso del nivel dos, la actitud positiva infundida por los profesores, disminuye a un $57.2 \%$ puesto que la relación entre estudiante-profesor tiende a ser más independiente, ya que las estudiantes comienzan a manifestar una total o parcial autonomía hacía los intereses que su propia identidad quiera revelar. Lo que implica que los profesores adquieran un mayor reto profesional al enseñar y motivar a sus estudiantes para la práctica de las matemáticas, resaltando que en estos grados algunos temas dejar de ser tan aplicables a la vida cotidiana, y resultan siendo para los estudiantes más mecánicos y menos prácticos,

Además, los métodos didácticos de enseñanza como también la estimulación para una mejoría en el desempeño académico, son menos frecuentes y significativos.

Análisis de la categoría compañeros. Los resultados nos presentan que los compañeros ejercen una actitud positiva frente a las experiencias matemáticas de los estudiantes. En el nivel uno, aunque esta influencia positiva es del $64 \%$ es menor, que en la de los estudiantes del nivel 2, quienes en un $77.4 \%$ manifiestan la influencia positiva que tienen sus compañeros,

Esto es ilustrado por Ángela, estudiante de grado undécimo, quien dice:

- Entrevistador: [...]iConsideras que tus amigas influyen en tu interés hacia las matemáticas?

- Ángela: Claro que sí.

- Entrevistador: ¿De qué manera?

- Ángela: Como ya estamos cerca de presentar el ICFES y las admisiones para la uni- versidad, nos ponemos a pensar que todo lo que nos han enseñado es importante para obtener un buen resultado.

- Entrevistador: Además de esto, iellas te dicen que te pueden servir en algo más?

- Ángela: Si, muchas veces cuando me va mal en matemáticas yo les digo que ya no quiero estudiar eso, pero ellas me recuerdan que así sea que yo vaya a estudiar medicina, las matemáticas en cualquier momento me van a ser útiles.

\section{Conclusiones}

La primera conclusión tiene que ver con la actitud negativa que aumenta del nivel al nivel 2 puesto que los estudiantes sienten que en el aula de clase el trabajo en matemáticas es mecánico y los ejercicios realizados no son significativos para la resolución de sus problemas cotidianos, por consiguiente se empieza a restar importancia a las matemáticas.

La segunda conclusión refleja en el nivel 1, al existir mayor motivación por parte del profesor los niños genera mayor confianza hacia sus habilidades en matemáticas, mientras que no ocurre lo mismo en el nivel 2. Además, éstos últimos, son conscientes que no tienen buenos hábitos de estudio, que no son responsables con sus deberes escolares y por esta razón tratar de aprender a último momento se dificulta mucho y trae como consecuencia baja confianza en su capacidad matemática.

La tercera conclusión, está en concordancia con la investigación de Bishop (2005), en relación a que el docente juega un papel importante en las clases de las matemáticas en la parte afectiva y le reconocen el esfuerzo por hacer los temas comprensibles, y por aplicar estrategias metodológicas que permitían la apropiación de los temas tratados. Más sin embargo, la investigación mostró el cambio significativo que existe en relación al uso de materiales didácticos, laboratorio de matemáticas, actividades lúdicas que en el nivel 1 son mucho más frecuentes y en el nivel 2 tienden a desaparecer. 
La cuarta conclusión, señala que las relaciones entre compañeros son muy importantes en las clases de matemáticas. Puesto que cuando se conforman lazos de amistad se observa que los estudiantes que obtienen mejores resultados explican y ayudan a sus amigos que tienen dificultades. Pero, los compañeros también pueden promover el fracaso, ya que su influencia distrae la atención en clase, trayendo con ellos dificultades a la hora de comprender los temas expuestos por el docente y esto a la vez conlleva al fracaso en la materia.

Una vez establecidas las conclusiones, se espera que éstas llamen la atención de los profesores de matemáticas en formación y en ejercicio para comprender mejor las interacciones sociales en el aula de clase y buscar cambios que permitan que el interés de los estudiantes hacia las matemáticas aumente día a día.

\section{Bibliografía}

Bishop, A. (2005). Las influencias sociales en la clase de matemáticas. En: A. Bishop, Aproximación sociocultural a la Educación Matemática. (p. 141-148). Cali: Universidad del Valle.

Blanco, H. (2006). La Etnomatemática en Colombia. Un programa en construcción. Revista BOLEMA - Boletim de EducaçãoMatemática , 19 (26), 49-75.

Gómez Chacón, I. (2000). Matemática emocional. Los afectos en el aprendizaje matemático.Madrid: Narcea.

Muñoz, F. \& Bravo, J. (2007). Estudio sobre las influencias que ejercen los padres, profesores y compañeros en la actitud de los estudiantes frente a las matemáticas, Trabajo de grado, Universidad de Nariño, Departamento de Matemáticas y Estadística, San Juan de Pasto.

Ursini, S., Sánchez, G., \& Orendain, M. (2004). Validación y confiabilidad de una escala de actitudes hacia las matemáticas y hacia las matemáticas enseñadas con computadora. Educación Matemática, 16(3), 5978. 